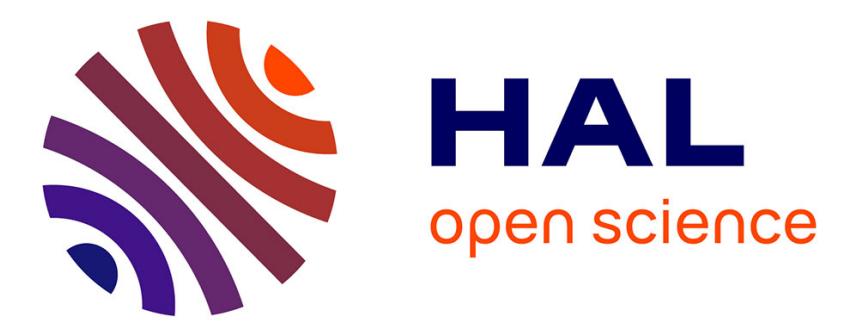

\title{
Coherent combining of optical parametric oscillators: challenges and experimental results
}

\author{
Pierre Bourdon, Rodwane Chtouki, Anne Durécu, Laurent Lombard,
} Christophe Planchat, Antoine Godard

\section{- To cite this version:}

Pierre Bourdon, Rodwane Chtouki, Anne Durécu, Laurent Lombard, Christophe Planchat, et al.. Coherent combining of optical parametric oscillators: challenges and experimental results. SPIE LASE 2021, Mar 2021, Online, United States. pp.116700H, 10.1117/12.2576878 . hal-03162389

\section{HAL Id: hal-03162389 \\ https://hal.science/hal-03162389}

Submitted on 8 Mar 2021

HAL is a multi-disciplinary open access archive for the deposit and dissemination of scientific research documents, whether they are published or not. The documents may come from teaching and research institutions in France or abroad, or from public or private research centers.
L'archive ouverte pluridisciplinaire HAL, est destinée au dépôt et à la diffusion de documents scientifiques de niveau recherche, publiés ou non, émanant des établissements d'enseignement et de recherche français ou étrangers, des laboratoires publics ou privés. 


\title{
Coherent combining of optical parametric oscillators: challenges and experimental results
}

\author{
P. Bourdon*, R. Chtouki, A. Durécu, L. Lombard, C. Planchat, A. Godard \\ ONERA, The French Aerospace Lab, BP 80100, 91123 Palaiseau Cedex, France
}

\begin{abstract}
Previous demonstrations of coherent combination of continuous-wave difference frequency generators (DFG) emitting at $3.4 \mu \mathrm{m}$ have proved that coherent beam combining (CBC) by active phase control could be useful for power scaling fiber-laser-pumped optical parametric oscillators (OPOs).

We developed an experiment using indirect phase control to demonstrate coherent combining of mid-infrared optical parametric oscillators. The main improvement from DFG combining is the enhancement in generated power and in conversion efficiency brought by the OPO cavity. But there's also an additional challenge: to be able to control the cavity modes of the OPO accurately to perform simultaneously real-time wavelength and phase control.

In this paper, we present the difficulties encountered to perform efficient coherent combining of mid-infrared emitting OPOs. We detail the different OPO cavity configurations we tested and compare theoretical assessment of their threshold with measurements.

The first results of coherent combining of continuous-wave OPOs are then given as well as the methods we use to couple the wavelength and phase control feedback loops.

Future work on the combining of pulsed OPOs is finally introduced as well as a comparison between pulsed laser combining and pulsed OPO combining limitations.
\end{abstract}

Keywords: coherent beam combining, nonlinear optics, difference frequency generation, optical parametric oscillator

\section{INTRODUCTION}

Coherent beam combining (CBC) techniques involving active phase control of the laser emitters have demonstrated their potential to power scale continuous wave $\mathrm{e}^{1,2}$ and pulsed $^{3}$ fiber lasers through coherent addition of the power emitted by multiple separate amplifiers. Using all-fiber components, very compact configurations of coherently combined fiber amplifiers can be designed, with demonstrated overall emitted power exceeding the kilowatt level ${ }^{2}$. Large numbers of combined fiber lasers, up to more than 100 , have also been demonstrated ${ }^{4-6}$, proving the scalability of this approach.

However, high power fiber lasers are only available within a finite set of wavelengths, mainly amongst $1 \mu \mathrm{m}, 1.5 \mu \mathrm{m}$ and $2 \mu \mathrm{m}$. Hopefully, nonlinear crystals offer the capability to convert these laser lines to access higher wavelengths in the midinfrared bands, for instance around $4 \mu \mathrm{m}$. But as with lasers, power scaling of frequency converters is limited, essentially by the damage threshold of the nonlinear medium. Coherent combining techniques would be extremely useful to power scale frequency converters beyond this damage threshold limit. Unfortunately, combination techniques are difficult to implement in this case, as active phase control requires fast phase modulators at the midinfrared wavelength emitted by the converter. Such components are not exactly "off-the-shelf" and are not as practical nor as fast as the allfiber modulators available at more standard wavelengths.

*pierre.bourdon@onera.fr; phone (+33)-1-80-38-63-82; fax (+33)-1-80-38-63-45; www.onera.fr 
Fortunately, taking advantage of the phase-matching relations involved in any frequency converting process, one can indirectly control the converted wave phase through control of the pump wave phase. When this pump wave is delivered by fiber amplifiers, such a frequency-converter coherent combining configuration can be achieved using standard offthe-shelf all-fiber phase modulators. Our team successfully applied this approach to perform efficient coherent combination of second harmonic generators and difference frequency generators ${ }^{7-9}$.

This DFG combining demonstration in the midinfrared delivered very low conversion efficiency, but it also proved that indirect phase control could be applied to non-degenerate third order nonlinear processes such as parametric amplification.

In this paper, we present the challenges of extending this indirect phase control technique to midinfrared OPO coherent combining.

An experimental demonstration of OPO CBC is also detailed. Results are presented and the technical issues encountered are explained. Design hints for coherent combining of continuous wave and pulsed OPOs are finally proposed.

\section{PRINCIPLE OF COHERENT COMBINING OF DFG CONVERTERS}

Coherent beam combining of non-degenerate three wave mixing nonlinear processes was first demonstrated on devices that didn't require a cavity to operate: difference frequency generators.

But the theoretical bases are identical to those of OPO CBC, as both experiments combine parametric amplifiers.

To achieve CBC of DFGs or OPOs, phase control of one of the converted waves, the signal or the idler waves, has to be performed.

Indirect phase control benefits from the phase-matching condition.

The electric field corresponding to each nonlinearly coupled wave, pump, signal and idler, can be written as:

$$
\widetilde{E}_{m}(z, t)=A_{m}(z) e^{j k_{m} z} e^{-j \omega_{m} t}+c . c
$$

where $m=s, i$ or $p$, and $A_{m}$ is the complex amplitude of the $\omega_{m}$ pulsation wave.

If we assume that the pump wave is not depleted when passing through the crystal, which is true on the first millimeters of the nonlinear crystal where parametric amplification is still weak, the coupled-amplitude equations describing difference frequency generation are:

$$
\left\{\begin{array}{l}
\frac{d A_{\mathrm{s}}(\mathrm{z})}{d z}=\frac{8 \pi j \omega_{\mathrm{s}}^{2} d_{e f f}}{k_{\mathrm{s}} c^{2}} A_{\mathrm{p}} A_{\mathrm{i}}^{*}(z) e^{j \Delta k z} \\
\frac{d A_{\mathrm{i}}(z)}{d z}=\frac{8 \pi j \omega_{\mathrm{i}}^{2} d_{e f f}}{k_{\mathrm{i}} c^{2}} A_{\mathrm{p}} A_{\mathrm{s}}^{*}(z) e^{j \Delta k z}
\end{array}\right.
$$

where $\Delta k=k_{p}-k_{S}-k_{i}$.

If we assume that the idler wave is not seeded, we can consider that $A_{i}(0)=0$. The initial value for the signal wave amplitude is noted $A_{s}(0)$. Thus, equations (2) can be solved using the notation $\kappa_{\mathrm{m}}=\frac{8 \pi \mathrm{j} \omega_{\mathrm{m}}^{2} \mathrm{~d}_{\mathrm{eff}}}{\mathrm{k}_{\mathrm{m}} \mathrm{c}^{2}}$ :

$$
\left\{\begin{array}{c}
A_{\mathrm{s}}(\mathrm{z})=A_{\mathrm{s}}(0) \cosh \left(\sqrt{\kappa_{\mathrm{s}} \kappa_{\mathrm{i}}^{*}\left|A_{\mathrm{p}}\right|^{2} z}\right) \\
A_{\mathrm{i}}(\mathrm{z})=\frac{\kappa_{\mathrm{i}} A_{\mathrm{p}}(0)}{\sqrt{\kappa_{\mathrm{s}} \kappa_{\mathrm{i}}^{*}\left|A_{\mathrm{p}}\right|^{2}}} A_{\mathrm{s}}^{*}(0) \sinh \left(\sqrt{\kappa_{\mathrm{s}} \kappa_{\mathrm{i}}^{*}\left|A_{\mathrm{p}}\right|^{2} z}\right)
\end{array}\right.
$$

These solutions correspond to a signal field that keeps its initial phase-offset, and is simply amplified by the interaction, while the idler wave phase-offset depends on both the pump wave's and the signal wave's.

For a bulk crystal with standard birefringent phase matching, one obtains the phase relation: $\varphi_{\mathbf{i}}=\varphi_{\mathbf{p}}-\varphi_{\mathbf{s}}+\frac{\pi}{2}$.

Similarly, in a perfect periodically poled crystal, the relation between phases of the pump, signal and idler waves can be obtained propagating the waves in the crystal domain by domain and step by step, leading do a similar relation: 


$$
\varphi_{\mathrm{i}}=\varphi_{\mathrm{p}}-\varphi_{\mathrm{s}}
$$

More generally, for a non perfect periodically poled crystal (for instance not exactly cut at a finite number of coherence lengths), or when phase matching is not perfect, an additional phase offset can appear in this relation, but this offset is fixed so that there's always a $\varphi_{\mathbf{i}}=\varphi_{0}+\varphi_{\mathrm{p}}-\varphi_{\mathrm{s}}$ relation between the 3 coupled waves in a DFG or parametric amplification process.

Consequently, controlling the pump wave phase gives control over the phase offset difference between the signal and idler waves. As we'll detail later on, this relation is enough for allowing indirect phase control of the idler wave with sole control of the pump wave phase.

\section{EXPERIMENTAL DEMONSTRATIONS OF DFG CBC}

We demonstrated experimentally the feasibility of such indirect phase control combining two DFG modules pumped by $1.064 \mu \mathrm{m} \mathrm{Yb}$ :fiber lasers delivering up to $15 \mathrm{~W}$ power each (see Fig. 1).

To fix and maintain the signal wavelength and phase, it is necessary to seed the DFGs with a signal seed at $1553 \mathrm{~nm}$ from a laser diode amplified by a commercial erbium-doped fiber amplifier split in two. Using this 1553-nm seeder, we are able to keep both DFG channels at a common and fixed signal wavelength.

The DFG nonlinear crystals are PPLN crystal from Covesion and their length is $L=20 \mathrm{~mm}$. With a 1064-nm pump wavelength and a seeded signal at $1553 \mathrm{~nm}$, quasi-phase matching in the PPLN crystal is obtained with a poling period of $30.49 \mu \mathrm{m}$ and at an operating temperature of $60^{\circ} \mathrm{C}$ for both crystals, generating an idler wave at $3.4 \mu \mathrm{m}$. The pump beams are focused down to $100 \mu \mathrm{m}$ waist in the PPLN crystals.

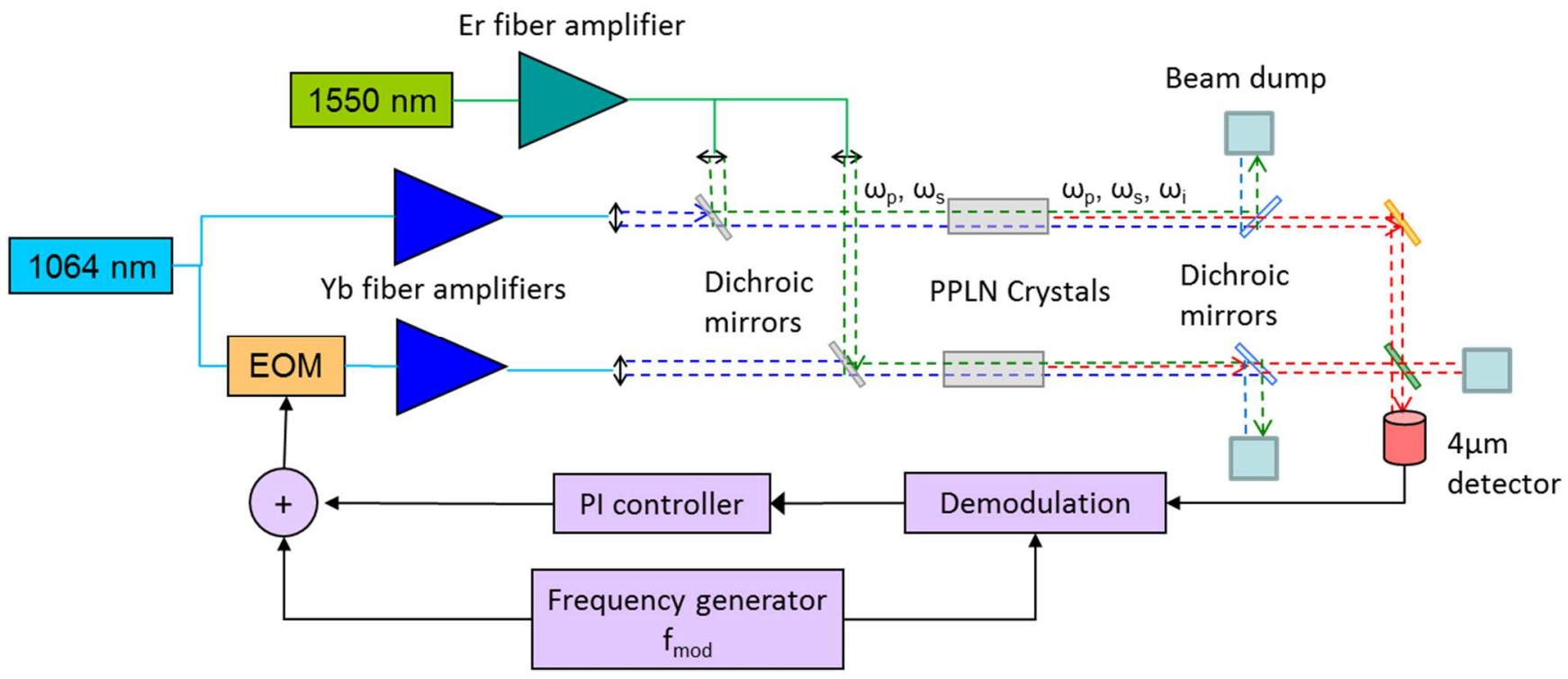

Figure 1. Schematics of the experimental set-up for demonstrating coherent combining of two 3.4- $\mu \mathrm{m}$ idler beams generated through DFG in PPLN crystals. Coherent combining is achieved by active phase-control of one of the pump waves.

Coherent combining of these DFG modules is achieved using the idler waves interference signal to close the feedback loop (see Fig. 2). Time-averaged combining efficiency is excellent: the residual phase error is $\lambda / 28 \mathrm{rms}$ for the idler wave.

This experiment is the proof that CBC of continuous wave DFG using indirect phase control is feasible, hence proving that $\mathrm{OPO} \mathrm{CBC}$ is also feasible as the nonlinear process involved in both cases is the same: parametric amplification.

The main difference between SHG CBC and DFG CBC is that simultaneous phase locking that has been proved feasible for SHG combination ${ }^{7}$, cannot be achieved so easily when combining non-degenerate three-wave mixing frequency conversion?. 
Even if seeded, the optical path difference between the two signal channels induces a phase difference that is not compensated for, and the signal waves are not phase-locked simultaneously when the idler waves are.

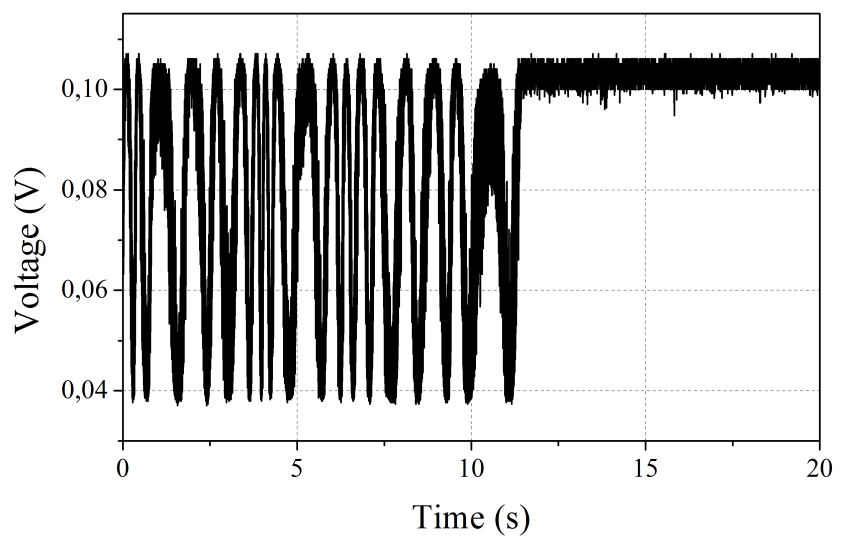

Figure 2. Time evolution of the interference signals of the idler beams when the phase control loop is open and then closed.

\section{OPTIMIZATION OF THE CW OPO DESIGN}

Unfortunately, the conversion efficiency of the DFG nonlinear process is extremely low, moreover when it is not enhanced by placing the nonlinear crystal in a cavity to benefit from conversion gain over multiple passes in the NL crystal. With $15 \mathrm{~W}$ pump power and watts of seeding power at $1553 \mathrm{~nm}$, the $3.4-\mu \mathrm{m}$ power generated was only in the few milliwatts level.

Optical parametric oscillators are operating using the same photon scheme of nonlinear process. However, they are more efficient sources in terms of conversion efficient as they benefit from having a cavity that enhances this efficiency. Part of the issue is also the length of the nonlinear crystal that was quite short for the DFG CBC experiment. So we provided longer PPLN crystals for the OPO, with $50 \mathrm{~mm}$ length.

Our first objective was to design an efficient continuous wave OPO with the capability to finely tune the signal wavelength so that we can achieve the same signal wavelength on multiple OPOs in order to be able to combine them. Singly resonant OPOs do not suffer from cavity mode clusters as doubly resonant ones do, and should see their signal wavelength be more smoothly tunable than DROPOs.

We used the same pump lasers as for the DFG CBC experiments with $15-20 \mathrm{~W}$ maximum power from these two fiber laser sources.

There are two main architectures for a continuous wave OPO, as can be seen in Fig. 3: ring and linear cavities can be used. A ring cavity generally offers lower threshold and the first challenge of developing cw OPOs for CBC was to lower as much as possible their threshold.

Lowering the threshold too much is not suitable either, as a cw OPO becomes unstable in terms of emitted power when pumped more than 3 times its threshold. Analytical estimates of continuous wave OPOs are available in the literature ${ }^{10}$.

We also developed our own numerical model to compute both the expected threshold and efficiency of the different cavity designs that we tested. Optimizing the design of the cavity in terms of pump, signal and idler beams overlap and minimizing the threshold simultaneously, different configurations with a theoretical threshold around $10 \mathrm{~W}$ were chosen and tested.

Another configuration of nested cavity doubly resonant OPO $\left(\mathrm{NesCOPO}^{11}\right)$ was also optimized with a threshold that could be as low as a few hundred milliwatts and fine wavelength tuning capability. 

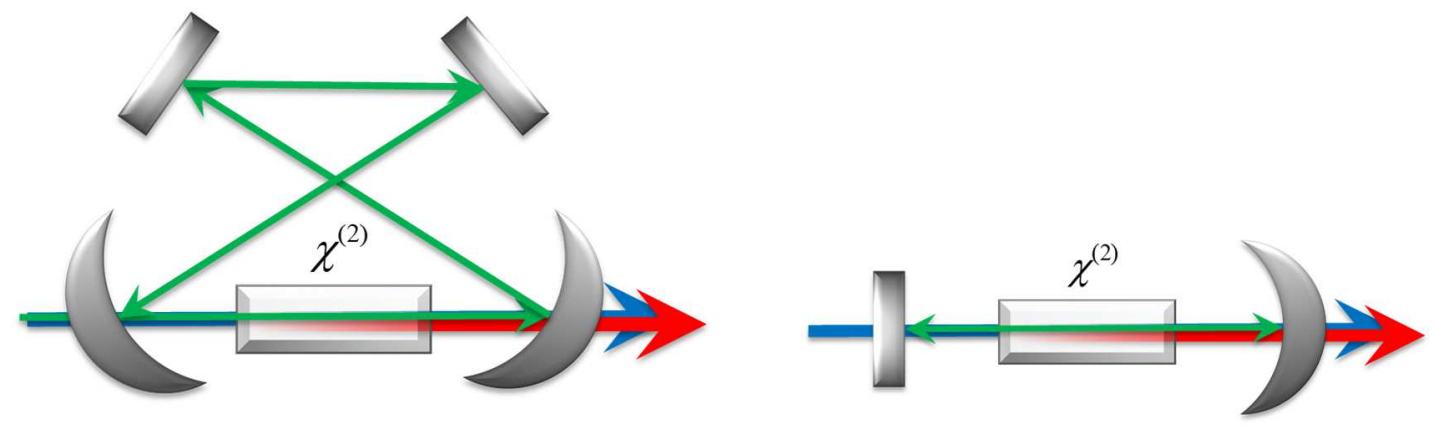

Figure 3. Cavity architectures of continuous wave OPOs.

One first observation we made is the extreme sensitivity of cw OPOs to the average reflectivity of their cavity mirrors. This average reflectivity must be as close as possible to $100 \%$. Even a few percent losses on the mirrors will result in a catastrophic drop in efficiency and increase in threshold for the cw OPO. The losses from the antireflection coatings on the nonlinear crystal faces must also be as low as possible.

We tested different ring cavity configuration but due to the higher number of mirrors in these configurations, the losses were to high to reach the OPO threshold, even with 20-W pump power.

The linear cavity configuration of Fig. 4 was the only one that allowed us to reach this threshold.

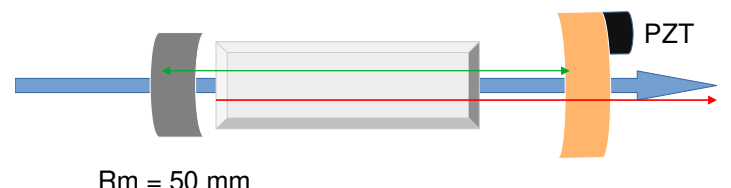

$\mathrm{Rm}=50 \mathrm{~mm}$

$\mathrm{Rm}=130 \mathrm{~mm}$

Figure 4. Linear cavity configuration with the lowest threshold that we decided to use eventually. The pump beam is in blue, the signal beam resonant in the cavity in green and the idler beam in red.

Numerical modelling of parametric amplification in this cavity demonstrated that to get a low threshold below $10 \mathrm{~W}$ pump power, it was necessary to use very high reflectivity cavity mirrors with less than $2 \%$ loss and a pump beam radius around $50-70 \mu \mathrm{m}$ in the NL crystal.

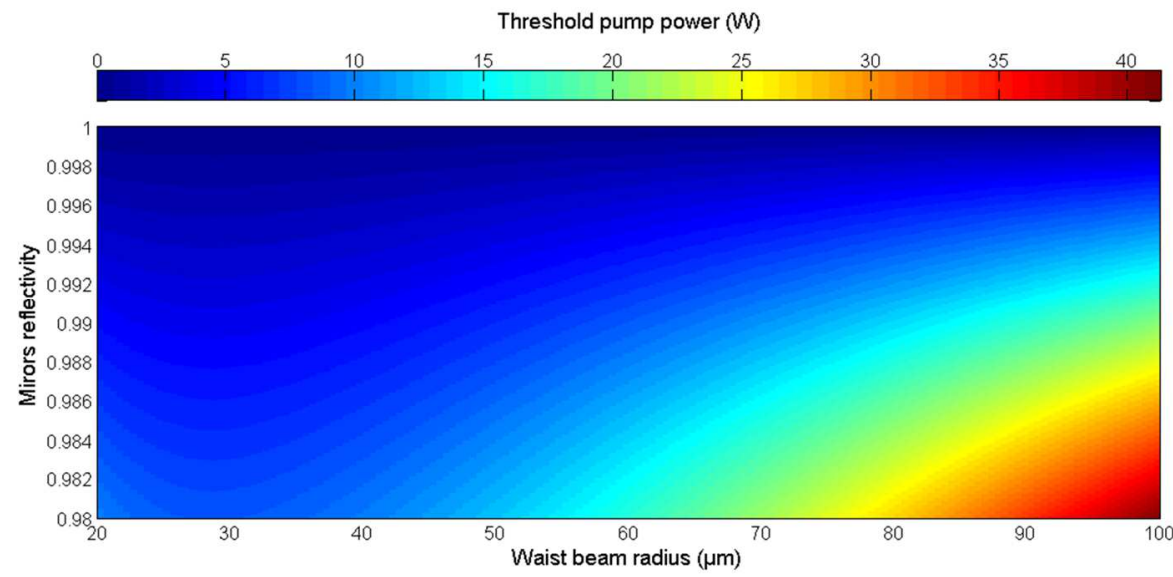

Figure 5. Numerical modelling of the linear cavity OPO threshold vs. pump beam waist radius in the NL crystal and average cavity mirrors reflectivity.

We characterized the reflectivity of the mirrors available in the laboratory to design the OPO cavity using a tunable laser source at $1.5 \mu \mathrm{m}$. An example of such mirror characterization is given in Fig. 6, proving that achieving an average mirror 
reflectivity of more than $98 \%$ is far from obvious, and limits the wavelength range for the signal to its lowest values, around $1480 \mathrm{~nm}$, in order to maximize the reflectivity of the cavity mirrors.

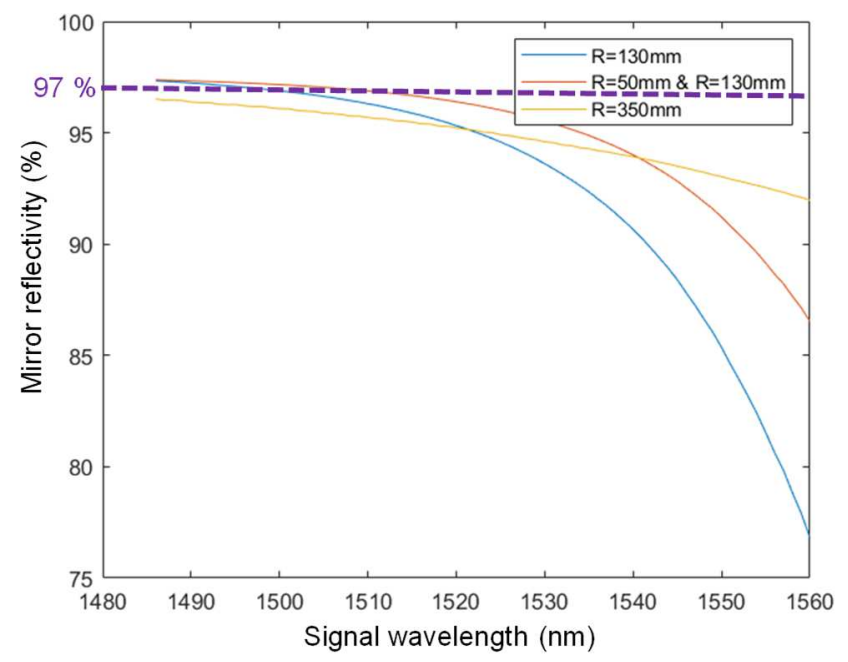

Figure 6. Evolution of the cavity mirrors reflectivity with the signal wavelength.

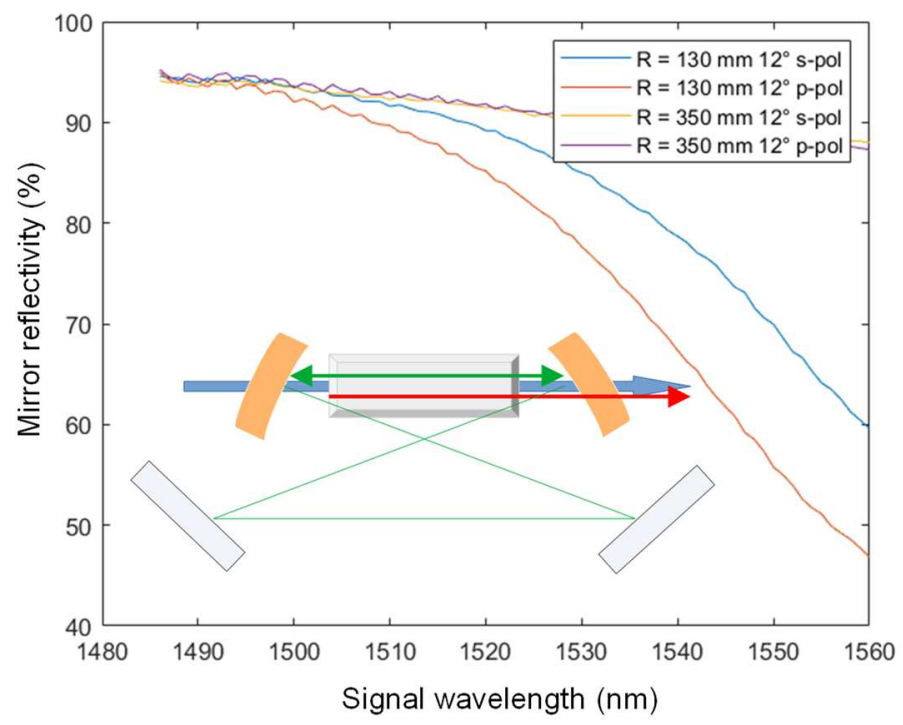

Figure 7. Evolution of the cavity mirrors reflectivity with the signal wavelength, for a ring cavity with the best mirrors available in the laboratory.

Fig. 7 shows why it was impossible to reach the OPO threshold with ring cavities, at least with the mirrors available in the laboratory to design this cavity.

Finally, with this choice of linear cavity, we met another issue due to the reflectivity of the cavity mirrors at $1.064 \mu \mathrm{m}$, the pump wavelength. This reflectivity, even if not very high, was sufficient to generate a parasitic Fabry-Perot for the pump wave. Hence, the intracavity pump power fluctuated making it even more difficult to align the OPO and reach the threshold.

However, despite these difficulties, we manage to obtain a significant boost in power efficiency using a cw OPO instead of DFG, as can be seen in Fig. 8. The OPO delivered idler in the hundreds of $\mathrm{mW}$ with the capability to reach the watt level if we had optimized mirrors for the cavity. 
The threshold is higher than the expected $5 \mathrm{~W}$ level, due to the $97 \%$ reflectivity of the cavity mirrors we used.

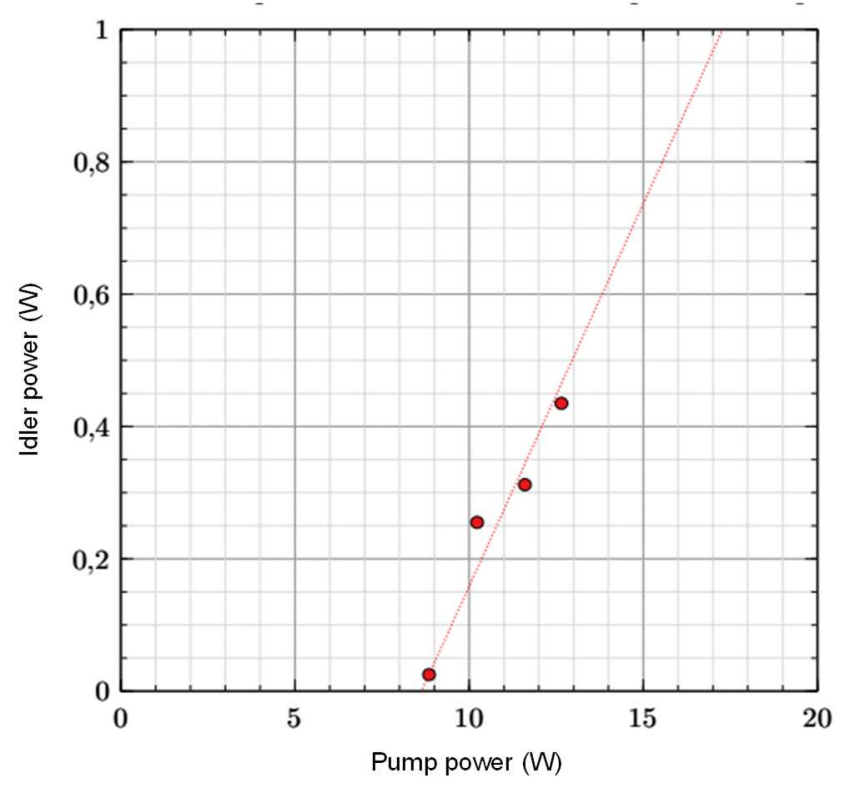

Figure 8. Idler power vs. pump power for the linear cavity OPO.

\section{COHERENT COMBINING OF OPO}

Next step was to duplicate this linear cavity OPO and prepare the coherent combining experiment (see Fig. 9).

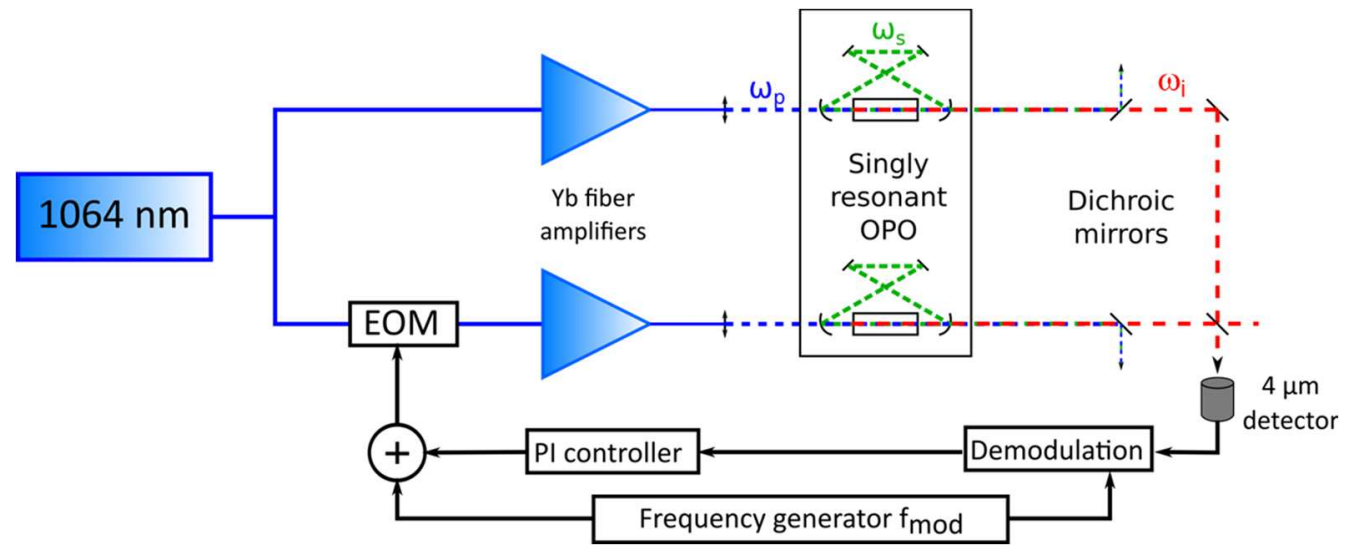

Figure 9. OPO CBC experimental setup. For the sake of making the figure easier to read, ring cavities are drawn for the OPO but we used the optimized linear cavity for both OPOs in the experiment.

Using a wavemeter and tuning finely, with 0.1 degree accuracy, the temperature of the second NL crystal, we were able to bring the second signal wavelength very close to the first one, sufficiently close to allow for fine adjustment of the second signal wavelength to the other signal wavelength value using cavity length tuning with a piezo-electric micropositioner.

Unfortunately, even with a singly-resonant OPO that should provide smooth tuning of the signal wavelength with the cavity length, we observed thermally induced longitudinal mode-hops that made it impossible to implement proper feedback on the signal wavelength through cavity length control.

Coherent combining of cw OPOs couldn't be achieved for the moment and this failed experiment proved that signal seeding should be added to verify that both OPOs emit the same signal and idler wavelengths. 


\section{CONCLUSION}

We developed an experiment of coherent combination of two continuous wave OPOs pumped at $1064 \mathrm{~nm}$ and emitting a signal wave around $1480 \mathrm{~nm}$ and an idler wave around $3785 \mathrm{~nm}$.

Optimization of the OPO conversion efficiency was achieved using a linear OPO cavity and minimizing the mirror losses below $3 \%$. The OPO threshold was between 8 and $9 \mathrm{~W}$ and $500 \mathrm{~mW}$ idler power was generated from this OPO.

In order to prevent fluctuations of the intracavity pump power, parasitic Fabry-Perot effects on the pump wave have to be mitigated by using high transmittance mirror for the pump beam.

After duplicating the OPO, coherent combining of OPOs was tested. Temperature tuning the crystal allowed to bring both signal wavelengths sufficiently close one to another that simple cavity length adjustment should be enough to equalize those wavelengths accurately.

However, unexpected longitudinal mode-hops rendered impossible such a feedback solution, proving that coherent combining of continuous wave OPOs requires proper seeding of the signal wavelengths to verify that the OPOs are operating at the same signal and idler wavelengths.

We started studying what we thought was a simpler case with continuous wave OPOs, as pulsed OPOs combination would definitely require seeding of the signal waves to provide a continuous wave signal inside the cavity to perform phase control between the short OPO pulses. This experimental study demonstrates that in fact, combining of continuous wave OPOs is not simpler than combining of pulsed OPOs. 


\section{REFERENCES}

[1] Flores, A., Shay, T.M., Lu, C.A., Robin, C., Pulford, B., Sanchez, A.D., Hult, D.W. and Rowland, K.B., "Coherent beam combining of fiber amplifiers in a kW regime," CLEO 2011 Conference, Paper CFE3 (2011).

[2] Shekel, E., Vidne, Y. and Urbach, B., " $16 \mathrm{~kW}$ single mode CW laser with dynamic beam for material processing," Proc. SPIE 11260, 1126021 (2020).

[3] Lombard, L., Azarian, A., Cadoret, K., Bourdon, P., Goular, D., Canat, G., Jolivet, V., Jaouën, Y. and Vasseur, O., "Coherent beam combination of narrow-linewidth $1.5 \mu \mathrm{m}$ fiber amplifiers in a long-pulse regime," Opt. Lett. 36(4), 523-525 (2011).

[4] Bourderionnet, J., Bellanger, C., Primot, J. and Brignon, A., "Collective coherent phase combining of 64 fibers. Opt. Express 19(18), 17053-17058 (2011).

[5] Su, R., Xi, J., Chang, H., Ma, Y., Ma, P., Wu, J., Jiang, M., Zhou, P., Si, L., Xu, X. and Chen, J., "Coherent combing of 60 fiber lasers using stochastic parallel gradient descent algorithm," ASSL 2019 Conference, Paper JW2A.1 (2019).

[6] Chang, H., Chang, Q., Xi, J., Hou, T., Su, R., Ma, P., Wu, J., Li, C., Jiang, M., Ma, Y. and Zhou, P., "First experimental demonstration of coherent beam combining of more than 100 fiber lasers," Phot. Research 8(12), 1943-1948 (2020).

[7] Odier, A., Durécu, A., Melkonian, J-M., Lombard, L., Lefebvre, M. and Bourdon, P., "Coherent combining of second-harmonic generators by active phase control of the fundamental waves," Opt. Lett. 42(16), 3201-3204 (2017)

[8] Odier, A., Durécu, A., Melkonian, J-M., Lombard, L., Lefebvre, M. and Bourdon, P., “Coherent combining of fiber-laser-pumped 3.4- $\mu$ m frequency converters," Proc. SPIE 10083, 1008319 (2017).

[9] Odier, A., Chtouki, R., Bourdon, P., Melkonian, J-M., Lombard, L., Godard, A., Lefebvre, M. and Durécu, A., "Coherent combining of mid-infrared difference frequency generators," Opt. Lett. 44(3), 566-569 (2019).

[10] Sutherland, R. L. (2003). Handbook of nonlinear optics, CRC press.

[11]Hardy, B. Berrou, A. Guilbaud, S. Raybaut, M. Godard, A. \& Lefebvre, M. (2011). Compact, single-frequency, doubly resonant optical parametric oscillator pumped in an achromatic phase-adapted double-pass geometry. Opt. Lett. 36(5), 678-680. 